\title{
Copeptin as a novel marker predicting prognosis of liver cirrhosis and its major complications
}

This article was published in the following Dove Press journal: Hepatic Medicine: Evidence and Research

\author{
Ahmed Khaled Tawfik' \\ Amal Helmy ${ }^{2}$ \\ Mohamed Yousef' \\ Sabry Abou-Saif' \\ Abdelrahman Kobtan' \\ Eman Asaad' \\ Sherief Abd-Elsalam \\ 'Department of Tropical Medicine \\ and Infectious Diseases, Faculty of \\ Medicine, Tanta University, Tanta, \\ Egypt; '2Department of Clinical \\ Pathology, Faculty of Medicine, Tanta \\ University, Tanta, Egypt
}

Objectives: The aim of the work was to assess the level of copeptin as a surrogate marker predicting the severity of liver diseases and its major complications.

Patients and methods: This was a cross-sectional study that included 40 patients and 10 controls and was performed in Tanta University Hospital between June 2016 and November 2016. The studied cases were divided into five groups: group I (10 patients): compensated cirrhosis; group II (10 patients): cirrhosis with gastrointestinal hemorrhage due to portal hypertension; group III (10 patients): cirrhosis with hepatorenal syndrome; group IV (10 patients): cirrhosis with liver cell failure; and group V (10 controls): normal healthy individuals.

Results: Regarding serum copeptin in the studied groups, copeptin showed a significant decrease in group I vs group II, group I vs group III, and group I vs group IV; and there was a significant increase in group II vs group III, group II vs group IV, group II vs control, group III vs control, and group IV vs control. No significance was detected between group I vs control and group III vs group IV.

Conclusions: Copeptin is a novel marker for the determination of prognosis of liver cirrhosis. There is significant association between serum level of copeptin and complications of liver cirrhosis.

Keywords: cirrhosis, hepatorenal syndrome, liver failure, varices, prognosis

\section{Introduction}

Cirrhosis is usually the final histological pathway whatever the underlying cause of liver diseases. ${ }^{1}$ There are many factors that can lead to liver cirrhosis and portal hypertension, which include viral hepatitis, ${ }^{2-5}$ alcohol abuse, sclerosing cholangitis, and common inborn errors of metabolism that include Wilson disease, hemochromatosis, and alpha-1 antitrypsin deficiency. ${ }^{6}$

One of the main complications of liver cirrhosis is portal hypertension. Clinically relevant complications of portal hypertension like the development of ascites and/or esophageal and gastric varices usually develop at a hepatic venous pressure gradient (HVPG) above $10 \mathrm{mmHg}{ }^{7}$

Portal hypertension is responsible for many critical complications like bleeding varices, ascites, hepatic encephalopathy, hepatorenal syndrome, and hepatic failure. The onset of these complications marks the deterioration of liver disease from a compensated to a decompensated stage. ${ }^{8}$

Ascites is one of the essential signs of hepatic decompensation and bad prognosis in patients with liver cirrhosis whatever the cause of hepatic injury. As soon as ascites develops, the incidence of mortality is set $50 \%$ at 2 years. $^{9-11}$
Correspondence: Sherief Abd-Elsalam Department of Tropical Medicine and Infectious Diseases, Faculty of Medicine, Tanta University, El Geish Street, Tanta, Gharbia Governorate, 35127, Egypt Tel +20 1095159522

Email Sherif_tropical@yahoo.com 
Refractory ascites occurs among about $20 \%$ of patients with ascites and is defined both as an unresponsiveness to salt restriction and high-dose diuretics and as a recurrence taking place rapidly within 4 weeks post-therapy, consistent with the American Association for the Study of Liver Diseases (AASLD) recommendations. ${ }^{12}$ Refractory ascites is usually related to poor prognosis and is a tough complication to deal with due to restricted treatment options for this special category of patients with chronic liver disease. ${ }^{13}$

Hepatorenal syndrome (HRS) is described as the development of renal failure in patients with advanced liver disease in the absence of other identifiable causes of renal failure. HRS can be of two types. Type 1 HRS develops unexpectedly and rapidly with a rise in serum creatinine to $>2.5 \mathrm{mg} / \mathrm{dL}$ in much less than 2 weeks. Additionally, it is usually preceded by a precipitating event, the most common being some bacterial infection including spontaneous bacterial peritonitis. Type 2 HRS is characterized by a slower development of renal dysfunction and commonly develops within the placing of refractory ascites. ${ }^{14}$

Hepatic encephalopathy (HE) is a primary trouble in patients with chronic liver diseases with neuropsychiatric manifestations ranging from sleep disturbance to deep coma. ${ }^{15}$

The most severe outcome of liver disease is the development of hepatic failure, which will only occur once $80 \%$ to $90 \%$ of hepatic functional capacity is removed. Hepatic failure is the endpoint of chronic hepatitis or alcoholic liver disease ending in cirrhosis. ${ }^{16}$

Copeptin is a 39-amino-acid glycopeptide with unknown physiological function or characteristic till now. However, copeptin is cosynthesized with vasopressin, which is likewise known as antidiuretic hormone, thereby immediately mirroring vasopressin levels. However, copeptin has the advantage of having more stability in the plasma and serum. ${ }^{17}$

Endotoxin and proinflammatory cytokines stimulates splanchnic arterial vasodilatation in cirrhotic patients leading to activation of the neurohumoral axis, which incorporates expanded activity of the sympathetic and renin-angiotensin-aldosterone systems, as well as the release of arginine vasopressin (AVP). ${ }^{18}$ It has been demonstrated that AVP concentrations increase greatly with worsening of liver function, and this important marker may additionally thus have a prognostic function. However, its measurement is not easy to carry out and now not routinely available. Copeptin, the pre-pro-AVP C-terminal fragment, is launched into the serum in equimolar quantities as AVP. For that reason, copeptin concentrations closely mirror the production of AVP, either in healthy subjects or in stressful situations inclusive of sepsis. The principal interest of copeptin is its stability in the serum, which is much more stable than AVP, thereby making it easier and more practical to measure. Moreover, its concentration increases much more than cortisol in the event of stress. ${ }^{19}$ The aim of this work was to evaluate copeptin as a novel marker in predicting the severity of liver diseases.

\section{Patients and methods}

This was a cross-sectional study that was performed on inpatients and outpatients of Tropical Medicine Department, Tanta University Hospital, between June 2016 and November 2016. The study included 40 patients and 10 controls.

The study took approval from the ethical evaluation board of Faculty of Medicine, Tanta University. A written informed consent was obtained from each patient enrolled in the study. The study protocol conforms to the ethical recommendations of the 1975 Declaration of Helsinki as contemplated in a priori approval by the institution's human research committee.

The studied cases were divided into 5 groups:

1. Group I (10 patients): Compensated cirrhosis.

2. Group II (10 patients): Cirrhosis with gastrointestinal (GI) hemorrhage due to portal hypertension. This was proved by upper endoscopy.

3. Group III (10 patients): Cirrhosis with HRS.

4. Group IV (10 patients): Cirrhosis with liver cell failure.

5. Group V (10 controls): Normal healthy individuals.

Diagnosis of cirrhosis depends on clinical, biochemical, and radiological criteria. Ultrasound diagnosis of cirrhosis was according to the criteria adapted by Awaya et al. ${ }^{20}$ The caudate lobe/right lobe ratio is above 0.65 , which can be indicative of liver cirrhosis, and its sensitivity, specificity, and accuracy reach $84 \%, 100 \%$, and $94 \%$, respectively. HRS was defined as renal failure in patients with severe liver disease in the absence of any identifiable renal pathology. ${ }^{21}$

According to Salerno et al, ${ }^{22}$ criteria for diagnosis of HRS were: 1) cirrhosis with ascites; 2) serum creatinine $>1.5$ $\mathrm{mg} / \mathrm{dL}$, no improvement of serum creatinine (a decrease in serum $<1.5 \mathrm{mg} / \mathrm{dL}$ ) after 2 days of diuretic withdrawal and volume expansion with albumin; 3) absence of shock; 4) no current or recent treatment with nephrotoxic drugs; and 5) absence of signs of parenchymal renal disease, as suggested by proteinuria ( $>500 \mathrm{mg} / \mathrm{dL}$ ) or hematuria ( $>50$ red blood cells per high-power field) and/or abnormal renal ultrasound. All cases were type II HRS. 


\section{Compensated cirrhosis}

Here, the liver function was maintained, with no vascular or parenchymatous signs of liver failure.

Patients with cirrhosis and its complications as hepatorenal failure, GI hemorrhage due to portal hypertension, or liver cell failure were enrolled in the study. Cases with 1) heart failure, 2) coronary insufficiency, 3) renal failure apart from HRS, 4) polyuria-polydipsia syndromes, and 5) hypotension or shock were excluded from the study.

Patients and controls were subjected to detailed history taking, thorough clinical examination, radiological investigations including abdominal ultrasonography, and laboratory investigations, including complete blood picture, random blood sugar, liver function tests (alanine aminotransferase [ALT], aspartate aminotransferase [AST], bilirubin, and serum albumin). Renal function tests included blood urea, serum creatinine, and urine analysis. Other investigations included estimation of prothrombin time, international normalized ratio (INR), and serum $\mathrm{Na}^{+}$. The Child-Pugh and Model for End-stage Liver Disease (MELD) score were also estimated (http://optn.transplant.hrsa. gov/resources/MeldPeldCalculator.asp?index=98). The ChildPugh is used to evaluate the prognosis of cirrhosis. Its main items include serum bilirubin, albumin, prothrombin time, presence of ascites, or HE. ${ }^{23}$ Measurement of serum copeptin by ELISA was done for all patients and controls.

\section{Specimen collection}

Five milliliters of venous blood sample was collected from each patient and control by venipuncture under complete aseptic conditions and was divided into three tubes as follows: blood was collected in tube containing EDTA for complete blood count; blood was collected in a tube containing citrate for prothrombin time and INR; the remaining blood was collected in plain tube, and serum was separated for estimation of random blood sugar, liver, and kidney function tests and serum $\mathrm{Na}^{+}$. The remaining part of the serum was preserved at $-20^{\circ} \mathrm{C}$ till the time of copeptin assay.

\section{Measurement of the level of serum copeptin}

Human copeptin was estimated by using ELISA kits (Catalog No. 201-12-5463) provided by Elabscience Company, Houston, TX, USA.

\section{Test principle}

The kit used a double-antibody sandwich (ELISA) to assay the level of human copeptin in samples. Copeptin was added to monoclonal antibody enzyme well, which was precoated with human copeptin monoclonal antibody followed by incubation. Copeptin antibodies were added, labeled with biotin, and combined with streptavidin-horseradish peroxidase to form immune complex; then, incubation was carried out and washed again to remove the uncombined enzyme. Then, chromogen solutions A and B were added, the color of the liquid changed to blue, and due to the effect of acid, the color finally became yellow. The color intensity and the concentration of copeptin in the sample were positively correlated.

\section{Statistical analysis}

The results were statistically analyzed by SPSS version 20 . Comparison of qualitative variables was performed with the chi-squared test. Student's $t$-test (or a nonparametric Mann-Whitney U-test when appropriate) was performed for comparisons of continuous data. A $P$-value $<0.05$ was considered statistically significant.

\section{Results}

Regarding liver functions in the studied groups (Table 1), total serum bilirubin showed a significant increase in group III vs group I and group III vs control. No significant changes were detected in group I vs group II, group I vs group IV, group I vs control, group II vs group IV, group II vs control, group III vs group IV, and group IV vs control. No significant difference was observed between the studied groups with respect to ALT and AST. There was a significant decrease of serum albumin in all groups compared with control, except group I compared with control.

Regarding Child classification in the studied groups (Table 2), group I included 10 cases with Child A. Group II included one case with Child A, five cases with Child B, and four cases with Child C. Group III included four cases with Child B and six cases with Child C. Group IV included six cases with Child B and four cases with Child C.

There was a significant difference among the four groups as regards Child score.

Serum copeptin in the studied groups showed a significant decrease in group I vs group II, group I vs group III, and group I vs group IV (Table 3 ). There was a significant increase in group II vs group III, group II vs group IV, group II vs control, group III vs control, and group IV vs control. There was no significant difference between group I vs control and group III vs group IV.

Copeptin showed a positive correlation with urea, creatinine, INR, and Child score and a negative correlation with $\mathrm{Na}^{+}$and albumin (Table 4).

Copeptin predicted liver disease at a cutoff value of $7 \mathrm{pmol} / \mathrm{L}$, with different sensitivity and specificity figures 
Table I Liver function tests among studied groups

\begin{tabular}{|c|c|c|c|c|c|c|c|c|c|c|}
\hline & & \multirow{2}{*}{\multicolumn{2}{|c|}{$\begin{array}{l}\text { Group I } \\
\text { Compensated } \\
\text { cirrhosis }\end{array}$}} & \multirow{2}{*}{\multicolumn{2}{|c|}{$\begin{array}{l}\text { Group II } \\
\text { Cirrhosis with } \\
\text { GI hemorrhage } \\
\text { due to PH }\end{array}$}} & \multirow{2}{*}{$\begin{array}{l}\text { Group III } \\
\text { Cirrhosis } \\
\text { with HRS }\end{array}$} & \multirow{2}{*}{$\begin{array}{l}\text { Group IV } \\
\text { Cirrhosis } \\
\text { with liver } \\
\text { failure }\end{array}$} & \multirow[t]{2}{*}{ Control } & \multirow[t]{2}{*}{$F$ test } & \multirow[t]{2}{*}{$P$-value } \\
\hline & & & & & & & & & & \\
\hline \multirow[t]{2}{*}{ Bilirubin (total) (mg/dL) } & Range & $0.5-1.9$ & & $0.8-8.4$ & & $0.5-10.8$ & $0.7-3.57$ & $0.5-1.66$ & 4.789 & $0.003^{a}$ \\
\hline & Mean \pm SD & $1.19 \pm 0.46$ & & $1.46 \pm 2.27$ & & $4.13 \pm 3.38$ & $1.75 \pm 0.98$ & $0.8 I \pm 0.35$ & & \\
\hline \multirow[t]{2}{*}{ Tukey's test } & PI & $\mathrm{P} 2$ & P3 & $\mathrm{P} 4$ & P5 & P6 & $\mathrm{P} 7$ & P8 & P9 & PIO \\
\hline & 0.566 & $0.010^{\mathrm{a}}$ & 0.964 & 0.992 & 0.296 & 0.915 & 0.307 & 0.053 & $0.003^{\mathrm{a}}$ & 0.804 \\
\hline \multirow[t]{2}{*}{$\mathrm{ALT}(\mathrm{U} / \mathrm{L})$} & Range & $25-44$ & & 18-77 & & $13-125$ & $\mathrm{II}-70$ & $15-40$ & 1.176 & 0.334 \\
\hline & Mean $\pm S D$ & $34.9 \pm 7.50$ & & $37.4 \pm 18.32$ & & $38.5 \pm 33.4 I$ & $38.6 \pm 17.88$ & $22.86 \pm 7.27$ & & \\
\hline \multirow[t]{2}{*}{ Tukey's test } & $\mathrm{PI}$ & $\mathrm{P} 2$ & P3 & P4 & P5 & P6 & P7 & P8 & P9 & PIO \\
\hline & 0.998 & 0.994 & 0.993 & 0.638 & 0.999 & 0.999 & 0.458 & 0.999 & 0.384 & 0.378 \\
\hline \multirow[t]{2}{*}{ AST (U/L) } & Range & $31-89$ & & $27-120$ & & $10-220$ & $26-180$ & $10-42$ & 2.170 & 0.088 \\
\hline & Mean \pm SD & $54.3 \pm 24.6$ & & $61.6 \pm 34.62$ & & $62.8 \pm 59.92$ & $77.3 \pm 45.49$ & $28.6 \pm 9.03$ & & \\
\hline \multirow{2}{*}{ Tukey's test } & PI & P2 & P3 & P4 & P5 & P6 & $\mathrm{P7}$ & P8 & P9 & PIO \\
\hline & 0.992 & 0.988 & 0.674 & 0.568 & 0.999 & 0.899 & 0.312 & 0.917 & 0.286 & 0.051 \\
\hline \multirow[t]{2}{*}{ Albumin (g/dL) } & Range & $3-3.8$ & & I.8-3.34 & & $1.8-2.6$ & I.4-2.8 & $3.5-3.97$ & 40.436 & $0.00 \mathrm{I}^{\mathrm{a}}$ \\
\hline & Mean \pm SD & $3.4 I \pm 0.26$ & & $2.7 I \pm 0.42$ & & $2.22 \pm 0.27$ & $2.26 \pm 0.48$ & $3.75 \pm 0.19$ & & \\
\hline \multirow[t]{2}{*}{ Tukey's test } & $\mathrm{PI}$ & $\mathrm{P} 2$ & P3 & $\mathrm{P} 4$ & P5 & P6 & $\mathrm{P7}$ & P8 & P9 & PIO \\
\hline & $0.00 I^{a}$ & $0.00 \mathrm{I}^{\mathrm{a}}$ & $0.00 \mathrm{I}^{\mathrm{a}}$ & 0.197 & $0.018^{a}$ & $0.036^{a}$ & $0.00 \mathrm{I}^{\mathrm{a}}$ & 0.999 & $0.00 I^{a}$ & $0.00 \mathrm{I}^{\mathrm{a}}$ \\
\hline
\end{tabular}

Notes: PI (group I and group II), P2 (group I and group III), P3 (group I and group IV), P4 (group I and control), P5 (group II and group III), P6 (group II and group IV), P7 (group II and control), P8 (group III and group IV), P9 (group III and control), and PIO (group IV and control). asignificant P-value $<0.05$.

Abbreviations: ALT, alanine aminotransferase; AST, aspartate aminotransferase; Gl, gastrointestinal; HRS, hepatorenal syndrome; PH, portal hypertension.

Table 2 Child score among studied groups

\begin{tabular}{|c|c|c|c|c|c|c|}
\hline \multicolumn{2}{|c|}{ Child score } & \multicolumn{4}{|l|}{ Group } & \multirow[t]{3}{*}{ Total } \\
\hline & & \multirow{2}{*}{$\begin{array}{l}\text { Group I } \\
\text { Compensated } \\
\text { cirrhosis }\end{array}$} & \multirow{2}{*}{$\begin{array}{l}\text { Group II } \\
\text { Cirrhosis with GI } \\
\text { hemorrhage due to PH }\end{array}$} & \multirow{2}{*}{$\begin{array}{l}\text { Group III } \\
\text { Cirrhosis } \\
\text { with HRS }\end{array}$} & \multirow{2}{*}{$\begin{array}{l}\text { Group IV } \\
\text { Cirrhosis with } \\
\text { liver failure }\end{array}$} & \\
\hline & & & & & & \\
\hline \multirow[t]{2}{*}{$A$} & $\mathrm{~N}$ & 10 & 1 & 0 & 0 & 11 \\
\hline & $\%$ & $100.0 \%$ & $10.0 \%$ & $0.0 \%$ & $0.0 \%$ & $27.5 \%$ \\
\hline \multirow[t]{2}{*}{ B } & $N$ & 0 & 5 & 4 & 6 & 15 \\
\hline & $\%$ & $0.0 \%$ & $50.0 \%$ & $40.0 \%$ & $60.0 \%$ & $37.5 \%$ \\
\hline \multirow[t]{2}{*}{ C } & $N$ & 0 & 4 & 6 & 4 & 14 \\
\hline & $\%$ & $0.0 \%$ & $40.0 \%$ & $60.0 \%$ & $40.0 \%$ & $35.0 \%$ \\
\hline \multirow[t]{2}{*}{ Total } & $\mathrm{N}$ & 10 & 10 & 10 & 10 & 40 \\
\hline & $\%$ & $100.0 \%$ & $100.0 \%$ & $100.0 \%$ & $100.0 \%$ & $100.0 \%$ \\
\hline Chi- & $\chi^{2}$ & 36.689 & & & & \\
\hline square & $P$-value & $0.001 *$ & & & & \\
\hline
\end{tabular}

Note: *Statistically significant.

Abbreviations: GI, gastrointestinal; HRS, hepatorenal syndrome; $\mathrm{PH}$, portal hypertension.

Table 3 Serum copeptin (pmol/L) among studied groups

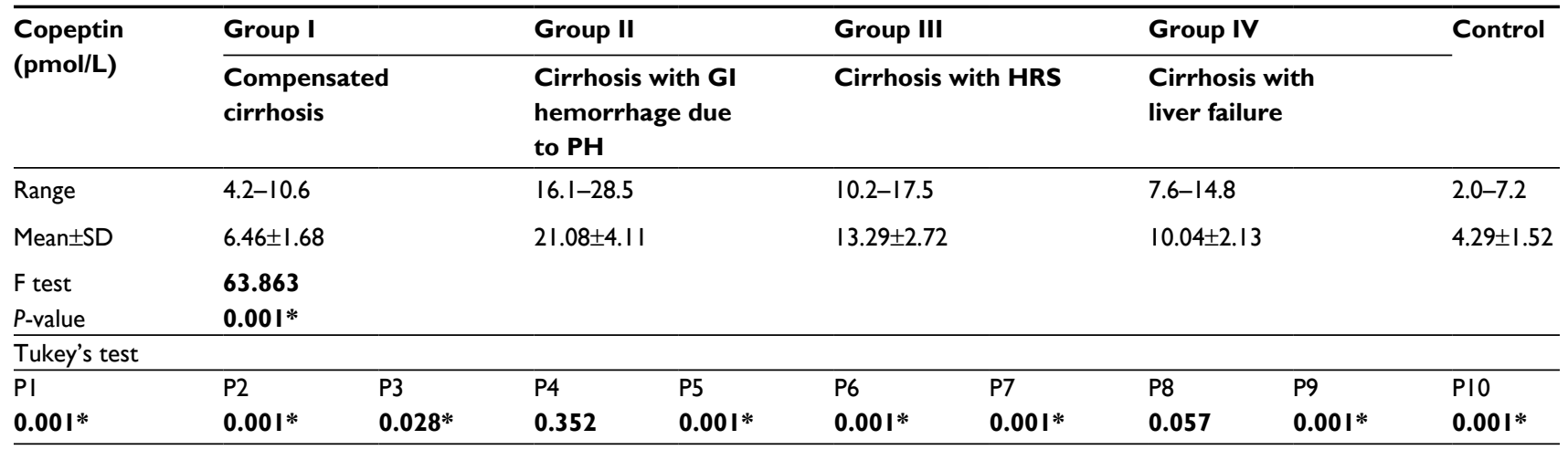

Notes: PI (group I and group II), P2 (group I and group III), P3 (group I and group IV), P4 (group I and control), P5 (group II and group III), P6 (group II and group IV), P7 (group II and control), P8 (group III and group IV), P9 (group III and control), and PIO (group IV and control). *Statistically significant.

Abbreviations: $\mathrm{Gl}$, gastrointestinal; HRS, hepatorenal syndrome; $\mathrm{PH}$, portal hypertension. 
Table 4 Linear correlation between copeptin and some parameters in studied groups

\begin{tabular}{lll}
\hline & \multicolumn{2}{l}{ Copeptin } \\
\cline { 2 - 3 } & $\boldsymbol{R}$ & $\boldsymbol{P}$ \\
\hline Creatinine & 0.598 & $0.036^{*}$ \\
Urea & 0.685 & $0.003^{*}$ \\
Bilirubin & 0.220 & 0.125 \\
Albumin & -0.863 & $0.00 I^{*}$ \\
INR & 0.730 & $0.002^{*}$ \\
$\mathrm{Na}$ & -0.810 & $0.00 I^{*}$ \\
Child score & 0.898 & $0.00 I^{*}$ \\
MELD & 0.493 & 0.066 \\
\hline
\end{tabular}

Note: *Statistically significant.

Abbreviations: INR, international normalized ratio; MELD, Model for End-stage Liver Disease.

Table 5 Sensitivity and specificity of copeptin at a cutoff value of $7 \mathrm{pmol} / \mathrm{L}$ in diagnosis of different groups

\begin{tabular}{lllll}
\hline & Sensitivity & Specificity & PPV & NPV \\
\hline Group I & 78 & 85 & 83 & 81 \\
Group II & 94 & 97 & 96 & 98 \\
Group III & 88 & 90 & 91 & 93 \\
Group IV & 82 & 83 & 89 & 87 \\
\hline
\end{tabular}

Notes: Group I: compensated cirrhosis; Group II: cirrhosis with gastrointestinal (Gl) hemorrhage due to portal hypertension; Group Ill: cirrhosis with HRS; Group IV: cirrhosis with liver cell failure. Group V ( 10 controls): Normal healthy individuals. Abbreviations: PPV, positive predictive value; NPV, negative predictive value.

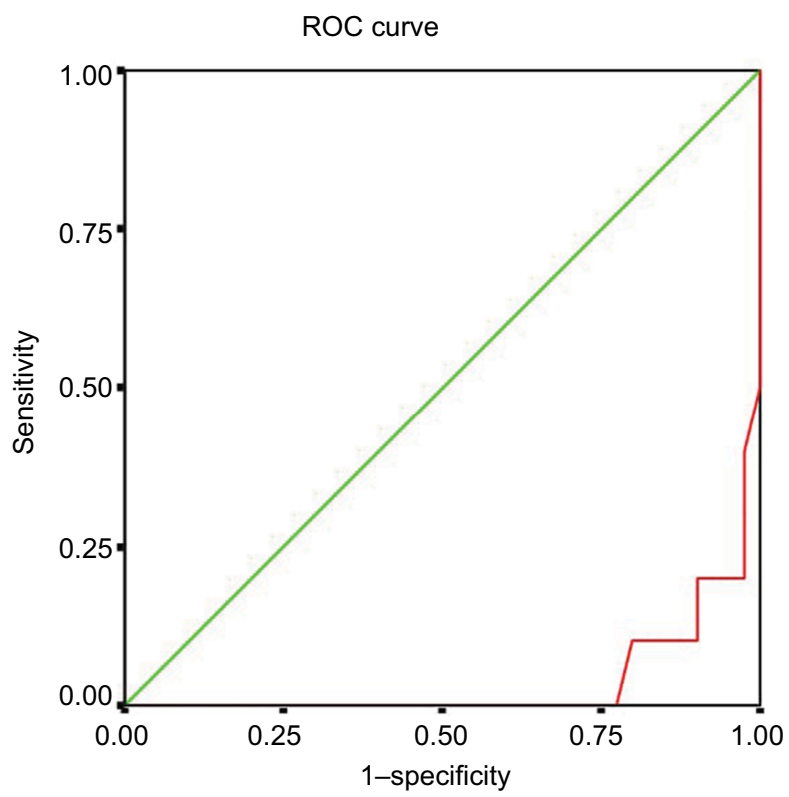

Figure I ROC of copeptin.

Abbreviation: ROC, receiver operating curve.

according to each group. The compensated cirrhosis group exhibited $78 \%$ sensitivity and $85 \%$ specificity. In GI hemorrhage group, $94 \%$ sensitivity and $97 \%$ specificity was observed. The HRS group exhibited $88 \%$ sensitivity and $90 \%$ specificity. The liver cell failure group showed $82 \%$ sensitivity and $83 \%$ specificity (Table 5, Figure 1).

\section{Discussion}

The present study found that copeptin was not elevated in compensated cirrhosis compared with control but was significantly elevated in GI hemorrhage group. This is in accordance with Solà et $a 1,{ }^{24}$ who found that the concentrations of copeptin, pro-adrenomedullin, and pro-A-type natriuretic peptide were elevated in patients with portal hypertension and HVPG above $12 \mathrm{mmHg}$. This can be due to the complex nature of the hyperdynamic disturbances in cirrhosis, involving a couple of organs and signaling pathways, suggesting that the hemodynamic impairment needs to affect peptide expression to some degree. Perhaps the hypovolemia associated with GI hemorrhage could have triggered AVP.

Recent trials have also found a strong correlation between copeptin and the degree of liver disease. ${ }^{25}$ The present study found that copeptin was significantly elevated in liver cell failure group. This is in accordance with Morgenthaler et al, ${ }^{19}$ who found that AVP concentrations increased with worsening of liver function, and this biological marker may thus have a prognostic function. Copeptin, the pre-pro-AVP $\mathrm{C}$-terminal fragment, is secreted into the serum in equimolar quantities to AVP.

Copeptin was also significantly elevated in GI hemorrhage, HRS, and liver cell failure groups vs compensated cirrhosis group. This is in accordance with Kimer et al, ${ }^{26}$ who found that patients with Child A cirrhosis had significantly lower concentrations of copeptin compared with Child C cirrhosis.

There was a significant positive correlation between the level of copeptin and serum creatinine. This is in accordance with Moreno et al. ${ }^{25}$

There was also a significant negative correlation between the copeptin level and serum $\mathrm{Na}^{+}$and albumin. This is in accordance with Solà et al, ${ }^{24}$ who found that AVP release from the neurohypophysis increases in parallel with the progression of cirrhosis and with circulatory dysfunction. Moreover, they have also suggested the important role of AVP in the development of hyponatremia. Also, this accords with Ball, ${ }^{27}$ who found in hypervolemic hyponatremia as in cirrhosis that the stimulation of AVP secretion was due to a secondary hemodynamic stimulus that showed a moderate increase of AVP.

Importantly, hyponatremia in cirrhosis is a frequent event well reflecting the severity of portal hypertension and independently associated with poor quality of life and mortality. 5,28

In our study, copeptin showed a significant positive correlation with Child score, which is in accordance with Moreno et al, ${ }^{25}$ who found that the copeptin concentrations were positively correlated with Child-Pugh score. 
The present study found no correlation between the level of copeptin and bilirubin. This is in accordance with Coenraad et al, ${ }^{29}$ who found that copeptin was not correlated with bilirubin concentration or INR.

There was a significant positive correlation in the level of copeptin with INR. This is not in accordance with Coenraad et al, ${ }^{29}$ who found that copeptin was not correlated with the use of diuretics, bilirubin concentration, or INR. This may be due to their study done on cases of cirrhosis in general and not on specific complication of cirrhosis.

Copeptin predicted liver disease at a cutoff value $7 \mathrm{pmol} / \mathrm{L}$ with different sensitivity and specificity figures according to each group. The compensated cirrhosis group exhibited $78 \%$ sensitivity and 85\% specificity. In GI hemorrhage group, 94\% sensitivity and $97 \%$ specificity was observed. The HRS group exhibited $88 \%$ sensitivity and $90 \%$ specificity. The liver cell failure group showed $82 \%$ sensitivity and $83 \%$ specificity. We detected that copeptin exhibited higher specificity and sensitivity in GI hemorrhage group and HRS group, indicating that it is a sensitive measure of hemodynamics.

Therefore, copeptin can be regarded as a novel prognostic marker of liver cirrhosis. There is a significant association between serum level of copeptin and complications of liver cirrhosis. Copeptin could be a marker for liver disease progression and follow-up. Being a marker for AVP, copeptin mirrors the hypovolemic changes due to liver failure. Copeptin concentrations increase along with the severity of liver disease as well as some of its complication.

The strength of this study is that this work adds an interesting aspect over the previous studies that are the strong correlation of copeptin with bleeding varices.

The major limitation of this study that it was conducted in a single center on relatively small number of patients.

We recommend that copeptin level could be used in the follow-up of cirrhosis and for anticipating its common complications. A further study is recommended using larger number of patients and longer duration to validate the present results.

\section{Disclosure}

The authors report no conflicts of interest in this work.

\section{References}

1. Tsochatzis EA, Bosch J, Burroughs AK. Liver cirrhosis. Lancet. 2014;383(9930):1749-1761.

2. Abd-Elsalam S, Sharaf-Eldin M, Soliman S, et al. Efficacy and safety of sofosbuvir plus ribavirin for treatment of cirrhotic patients with genotype 4 hepatitis $\mathrm{C}$ virus in real-life clinical practice. Arch Virol. 2018;163(1):51-56.
3. Ahmed OA, Kaisar HH, Hawash N, et al. Efficacy of sofosbuvir plus ribavirin with or without peginterferon-Alfa in treatment of a cohort of Egyptian patients with hepatitis C virus infection. Infect Disord Drug Targets. 2017;17(2):95-100.

4. Ahmed OA, Elsebaey MA, Fouad MHA, et al. Outcomes and predictors of treatment response with sofosbuvir plus daclatasvir with or without ribavirin in Egyptian patients with genotype 4 hepatitis $C$ virus infection. Infect Drug Resist. 2018;11:441-445.

5. Ahmed OA, Kaisar HH, Badawi R, et al. Efficacy and safety of sofosbuvir-ledipasvir for treatment of a cohort of Egyptian patients with chronic hepatitis C genotype 4 infection. Infect Drug Resist. 2018;11:295-298.

6. Zhou GW, Tao ZY, Peng CH, Li HW. Reasonable choice of surgical procedures for patients with portal hypertension. Hepatobiliary Pancreat Dis Int. 2003;2(3):330-333.

7. Groszmann RJ, Abraldes JG. Portal hypertension: from bedside to bench. J Clin Gastroenterol. 2005;39(4 Suppl 2):S125.

8. D'Amico G, Garcia-Tsao G, Pagliaro L. Natural history and prognostic indicators of survival in cirrhosis: a systematic review of 118 studies. J Hepatol. 2006;44(1):217-231.

9. Guevara M, Cárdenas A, Uriz J, Ginès P. Prognosis in patients with cirrhosis and ascites. In: Ginès P, Arroyo V, Rodés J, Schrier RW, editors. Ascites and Renal Dysfunction in Liver Disease: Pathogenesis, Diagnosis and Treatment. Malden: Blackwell; 2005:260-270.

10. Sharaf-Eldin M, Bediwy AS, Kobtan A, et al. Pigtail catheter: a less invasive option for pleural drainage in Egyptian patients with recurrent hepatic hydrothorax. Gastroenterol Res Pract. 2016;2016: 4013052-4013055.

11. Elfert A, Abo Ali L, Soliman S, Ibrahim S, Abd-Elsalam S. Randomizedcontrolled trial of rifaximin versus norfloxacin for secondary prophylaxis of spontaneous bacterial peritonitis. Eur J Gastroenterol Hepatol. 2016;28(12):1450-1454.

12. Runyon BA. Introduction to the revised American Association for the Study of Liver Diseases Practice Guideline management of adult patients with ascites due to cirrhosis 2012. Hepatology. 2013;57(4):1651-1653.

13. Bhogal H, Sanyal AJ. Treatment of refractory ascites. Clin Liver Dis. 2013;2(3):140-142.

14. Wong F, Nadim MK, Kellum JA, et al. Working Party proposal for a revised classification system of renal dysfunction in patients with cirrhosis. Gut. 2011;60(5):702-709.

15. Häussinger D, Kircheis G, Fischer R, Schliess F, vom Dahl S. Hepatic encephalopathy in chronic liver disease: a clinical manifestation of astrocyte swelling and low-grade cerebral edema? J Hepatol. 2000;32(6): 1035-1038.

16. Yee HF, Lidofsky SD. Acute liver failure. 7th ed. In: Feldman M, Friedman LS, Sleisenger MH, editors. Sleisenger and Fordtran's Gastrointestinal and Liver Disease: Pathophysiology, Diagnosis, Management. Philadelphia: Saunders; 2002:567-574.

17. Katan M, Müller B, Christ-Crain M. Copeptin: a new and promising diagnostic and prognostic marker. Crit Care. 2008;12(2):117-118.

18. Chikanza IC, Petrou P, Chrousos G. Perturbations of arginine vasopressin secretion during inflammatory stress. Pathophysiologic implications. Ann N Y Acad Sci. 2000;917:825-834.

19. Morgenthaler NG, Struck J, Jochberger S, Dünser MW. Copeptin: clinical use of a new biomarker. Trends Endocrinol Metab. 2008;19(2):43-49.

20. Awaya H, Mitchell DG, Kamishima T, Holland G, Ito K, Matsumoto T. Cirrhosis: modified caudate-right lobe ratio. Radiology. 2002;224(3):769-774.

21. Angeli P, Merkel C. Pathogenesis and management of hepatorenal syndrome in patients with cirrhosis. J Hepatol. 2008;48(Suppl 1): S93-S103.

22. Salerno F, Gerbes A, Ginès $\mathrm{P}$, Wong F, Arroyo V. Diagnosis, prevention and treatment of hepatorenal syndrome in cirrhosis. Gut. 2007;56(9): $1310-1318$.

23. Cholongitas E, Papatheodoridis GV, Vangeli M, Terreni N, Patch D, Burroughs AK. Systematic review: The model for end-stage liver diseaseshould it replace Child-Pugh's classification for assessing prognosis in cirrhosis? Aliment Pharmacol Ther. 2005;22(11-12):1079-1089. 
24. Solà E, Moreira R, Rodriguez E, et al. 605 Plasma copeptin levels are increased in cirrhosis and correlate with hyponatremia and circulatory dysfunction. J Hepatol. 2013;58:S247-S407.

25. Moreno JP, Grandclement E, Monnet E, et al. Plasma copeptin a possible prognostic marker in cirrhosis. Liver Int. 2013;33(6) 843-851.

26. Kimer N, Goetze JP, Bendtsen F, Møller S. New vasoactive peptides in cirrhosis: organ extraction and relation to the vasodilatory state. Eur J Clin Invest. 2014;44(5):441-452.
27. Ball SG. Vasopressin and disorders of water balance: the physiology and pathophysiology of vasopressin. Ann Clin Biochem. 2007;44(Pt 5):417-431.

28. Kim WR, Biggins SW, Kremers WK, et al. Hyponatremia and mortality among patients on the liver-transplant waiting list. $N$ Engl J Med. 2008;359(10):1018-1026.

29. Coenraad MJ, de Rooij BJ, Verbruggen L, van Hoek B, van der Reijden JJ, Verspaget HW. 619 Copeptin; An independent prognostic factor in cirrhosis? J Hepatol. 2012;56(2):S245-S246.
Hepatic Medicine: Evidence and Research

\section{Publish your work in this journal}

Hepatic Medicine: Evidence and Research is an international, peerreviewed, open access journal covering all aspects of adult and pediatric hepatology in the clinic and laboratory including the following topics: Pathology, pathophysiology of hepatic disease; Investigation and treatment of hepatic disease; Pharmacology of drugs used for the treatment

\section{Dovepress}

of hepatic disease. Issues of patient safety and quality of care will also be considered. The manuscript management system is completely online and includes a very quick and fair peer-review system, which is all easy to use. Visit http://www.dovepress.com/testimonials.php to read real quotes from published authors.

Submit your manuscript here: https://www.dovepress.com/hepatic-medicine-evidence-and-research-journal 